\title{
Configurational fluctuations and flavin-substrate interactions in the flavoenzyme ThyX studied by time- and spectrally resolved fluorescence
}

\author{
S. P. Laptenok, L. Bouzhir-Sima, H. Myllykallio, U. Liebl, and M. H. Vos \\ Laboratory for Optics and Biosciences, CNRS UMR7645, INSERM U696, Ecole Polytechnique, \\ 91128 Palaiseau, France
}

\begin{abstract}
Femtosecond-resolved fluorescence of bacterial thymidilate synthase using a Kerr-gate based setup identifies a close-by tyrosine involved in flavin fluorescence quenching, shows that the substrate dUMP acts as a strong quencher itself and highlights functional configurational flexibility
\end{abstract}

\section{Introduction}

In flavoproteins, interaction of the flavin cofactor with the protein environment is known to modulate the lifetime of the flavin fluorescence on the femtosecond to picosecond timescale, in particular due to quenching by photooxidation of nearby aromatic residues [1]. Thus, fluorescence decay is a sensitive probe of the protein configuration.

Thymidylate synthase ThyX is a flavo-enzyme discovered a decade ago [2] that is essential for de novo synthesis of the DNA base thymine in many bacteria. Its catalytic mechanism is very different from the one in humans and therefore considered a promising antimicrobial target. The flavincontaining catalytic site interacts with three different substrates and is speculated to be highly flexible to achieve its function.

In order to study configurational dynamics in the active site, we used a newly developed Kerr-gate based fluorescence spectrometer with full spectral resolution to investigate spectro-temporal properties of FAD (flavin adenine dinucleotide) fluorescence in wild-type (WT) and genetically modified ThyX from the thermophilic bacterium Thermotoga maritima.

\section{Materials and methods}

\subsection{Time-resolved fluorescence setup}

Our setup based on a Kerr shutter [3] allows measuring fluorescence spectra with a resolution of $\sim 100 \mathrm{fs}$ and up to the nanoseconds timescale. The setup, based on a $1 \mathrm{kHz}$ Ti:Saph system, is outlined in Fig. 1. Slices of the total emission are gated by an $800-\mathrm{nm}$ pulse that rotates the polarization in a non-linear optically active medium (supracil or $\mathrm{CS}_{2}$ ) placed between crossed polarisers and subsequently spectrally dispersed. Using spherically symmetric Cassegrain-type reflective objectives between the pair of thin high-contrast polarisers (Moxtek PFU04C) allows minimising temporal dispersion and depolarization. By using different Kerr media it is possible to 
vary width and efficiency of the gate, and thereby to adapt the ratio of the gated to the non-gated fluorescence to the timescale of the experiment.

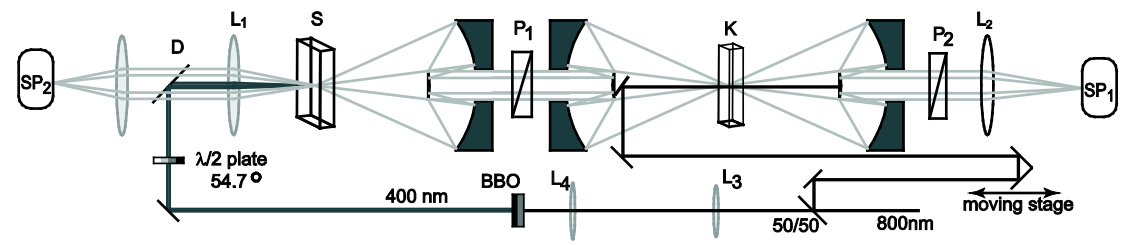

Fig. 1. Schematic representation of the setup. $\mathrm{S}$ - sample, $\mathrm{K}$ - Kerr cell, $\mathrm{P}_{1}, \mathrm{P}_{2}-$ crossed polarisers, $\mathrm{O}_{1}, \mathrm{O}_{2}$, $\mathrm{O}_{3}$ - reflective objectives, $\mathrm{SP}_{1}$ - spectrometer connected to cooled $\mathrm{CCD}$ for gated fluorescence detection, $\mathrm{SP}_{2}$ - reference spectrometer, $\mathrm{D}$ - dichroic mirror.

\subsection{Data analysis}

To describe ThyX fluorescence kinetic we combined datasets measured with two time-resolutions, and analyze them simultaneously using a parallel model, in which $n_{\text {comp }}$ excited species decay monoexponentially in parallel with $n_{\text {comp }}$ rate constants $k_{i}$. In this procedure, spectral shapes are left unconstrained yielding Decay Associated Spectra (DAS). For more details about the use of compartmental models for global of time-resolved spectroscopy data see reviews by van Stokkum and co-workers [4]. Global analysis was performed using open-source software called Glotaran, a problem solving environment for fitting superposition models to multi-dimensional data (http://glotaran.org)[5].

\section{Results and discussion}

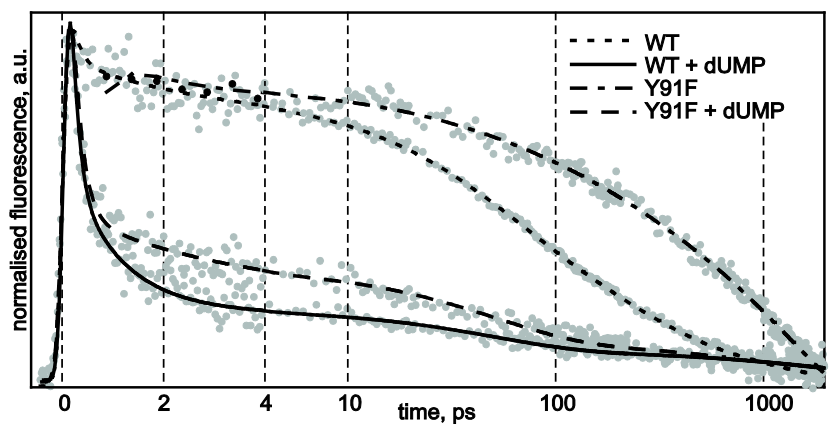

Fig 2. Fluorescence of ThyX at $520 \mathrm{~nm}$ upon $400 \mathrm{~nm}$ excitation. Time axe is in lin-log scale (until $4 \mathrm{ps}-$ linear scale after logarithmic scale)

In the oxidized WT, ThyX enzyme, the fluorescence predominantly decays much faster than the intrinsic nanosecond ( $\sim 5 \mathrm{~ns})$ decay of FAD. As in many other flavoproteins we assign this quenching to electron transfer from closeby aromatic residues to the excited flavin. The spectra decay in a remarkably multiphasic manner, spanning timescales from $\sim 1$ ps to $\sim 1$ ns. This indicates the presence of a broad range of configurations that do not fully interconvert on the timescale of the experiment. Up to $\sim 200 \mathrm{ps}$ a red-shift of the spectrum is observed, indicative of relaxation processes. The spectral shape remains unchanged after $\sim 200 \mathrm{ps}$ and is significantly different from that of FAD in solution, implying that free FAD does not contribute (fig 3A). According to the crystal structure of ThyX [6] Tyr91 is the closest aromatic residue to the FAD cofactor, and therefore a likely candidate as a main fluorescence quencher. Indeed, in the mutant Y91F where this Tyr was mutated to ET-incapable but sterically similar Phe, fluorescence decay kinetics are much slower compared to WT (fig 2). However in both cases a fast decay process in the order of few picoseconds is present in 
the data (fig 3B). The fluorescence decay of the Y91F become more homogenous comparing to WT, i.e, the dominating component (1.3 ns, dash-dot DAS, fig 3B) has much higher amplitude than others, however presence of significant amount of faster components suggests that conformation flexibility is maintained in the mutant. The presence of a fast phase in the fluorescence that is almost identical in the WT and Y91F indicating that that component represents process different from $e^{-}$transfer; can be probably assigned to higher excited state fluorescence. Differences in the shape of the component, with the life time of 35/30ps (dashed DAS, fig $3 \mathrm{AB}$ ), can be attributed to a different "weight" of the relaxation process with respect to overall decay on the tens of picoseconds timescale. ThyX converts the substrate deoxyuridine monophosphate (dUMP) to thymidylate (dTMP). For this reaction the enzyme accepts a methyl group from a (second) folate substrate and electrons from the flavin-cofactor after being pre-reduced by NADPH. In the absence of the other substrates, dUMP tightly binds close to oxidized FAD [6] leading to dramatic quenching of FAD fluorescence (fig 2). We resolved that the main decay occurs as fast as $\sim 200$ fs. Slower phases are also observed, indicating that substantial configurational flexibility is maintained. While a direct interaction between dUMP and the FAD cofactor may be at the origin of the very fast decay, the crystal structure also suggests that dUMP-binding brings the Tyr91 residue closer to the flavin. We therefore used the dUMP-bound Y91F ThyX mutant enzyme to investigate the origin of the very fast decay. As in this complex a dominant 200 -fs phase is also present, we conclude that dUMP quenches flavin fluorescence, presumably by acting as an electron donor to the excited FAD* state. This a priori unexpected result provides the perspective of detailed studies of molecular dynamics in the enzyme-substrate complex in solution and in the presence of other substrates or substrate analogs.
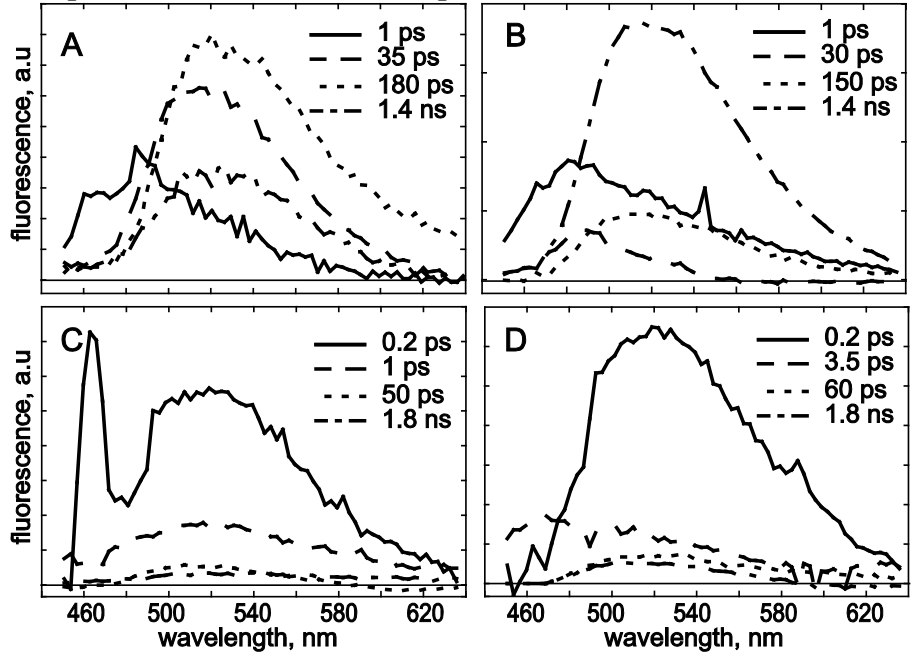

Fig. 3. DAS of ThyX obtained after global analysis of the WT (A) and mutant Y91F (B), WT-dUMP complex (C) Y91F-dUMPcomplex (D). The corresponding lifetimes are given in the figure. Excitation 400nm.

\section{References}

1. N. Nunthaboot, F. Tanaka, S. Kokpol, H. Chosrowjan, S. Taniguchi, N. Mataga, J. Phys. Chem. B 112, 41 (2008).

2. H. Myllykallio, G. Lipowski, D. Leduc, J. Filee, P. Forterre, U. Liebl, Science 297, 5578 (2002).

3. S. Arzhantsev M. Maroncelli, Applied Spectroscopy, 59 (2005)

4. I.H.M. van Stokkum, D.S. Larsen, R. van Grondelle, BBA, 1657, 2-3 (2004).

5. J.J. Snellenburg, S.P. Laptenok, R. Seger,K.M. Mullen, I.H.M van Stokkum, Journal of Statistical Software, 493 (2012)

6. I.I. Mathews, A.M. Deacon, J.M. Canaves, D. McMullan, S.A. Lesley, S. Agarwalla, P. Kuhn, Structure, 11, 6 (2003). 